\title{
Polymicrogyria with optic nerve hypoplasia
}

INSERM

\section{Source}

INSERM. (1999). Orphanet: an online rare disease and orphan drug data base.

Polymicrogyria with optic nerve hypoplasia. ORPHA:250972

Polymicrogyria with optic nerve hypoplasia is a rare genetic syndrome with central nervous system malformations characterized by severe developmental delay, neonatal hypotonia, seizures, optic nerve hypoplasia and distinct central nervous system malformations including extensive bilateral polymicrogyria, dysplastic or absent corpus callosum and malformed brainstem with loss of demarcation of the pontomedullary junction. 